\title{
DETERMINAÇÃO DE Mn E Zn EM ARROZ EMPREGANDO ESPECTROMETRIA DE FLUORESCÊNCIA DE RAIOS X DE ENERGIA DISPERSIVA
}

\author{
Alete Paixão Teixeira, Cristina M. Quintella e Maria das Graças Andrade Korn* \\ Instituto de Química, Universidade Federal da Bahia, Campus de Ondina, 40170-115 Salvador - BA, Brasil \\ Andrea P. Fernandes \\ Instituto de Química e Biotecnologia, Universidade Federal de Alagoas, Campus A. C. Simões, 57072-970 Maceió - AL, Brasil \\ Martha T. P. O. Castro \\ Universidade Federal Rural do Rio de Janeiro, 23890-000 Seropédica - RJ, Brasil
}

Recebido em 1/9/11; aceito em 21/12/11; publicado na web em 23/3/12

\begin{abstract}
DETERMINATION OF Mn AND Zn IN RICE BY ENERGY DISPERSIVE X-RAY FLUORESCENCE SPECTROMETRY. A simple, fast and inexpensive method was developed to determine essential elements in pellets of rice samples using energy dispersive X-ray fluorescence spectrometry (EDXRF). The accuracy and precision were evaluated using Standard Reference Material (rice flour NIST 1568a), and yielding relative standard deviation below $5 \%$. The paired t-test showed good agreement within $95 \%$ confidence values. The detection limits $(3 \sigma)$ of $\mathrm{Mn}$ and $\mathrm{Zn}$ were 5.1 and $2.2 \mathrm{mg} \mathrm{kg}^{-1}$, respectively. The proposed method proved to be effective when used to determine $\mathrm{Mn}$ and $\mathrm{Zn}$ in commercial samples of rice without go by stage of decomposition.
\end{abstract}

Keywords: rice; essential elements; EDXRF.

\section{INTRODUÇÃO}

O arroz (Oryza sativa, Linn.) é um dos cereais mais consumidos no mundo. Mesmo não sendo uma fonte rica em nutrientes inorgânicos, sua contribuição para a ingestão diária recomendada (IDR) de Mn e Zn é de, respectivamente, 50 e 19\%, tomando-se como base o consumo médio diário aproximado de $108 \mathrm{~g}$ per capita no período de 1964 a 2000 no Brasil. ${ }^{1-3}$

Elementos traço, tais como Mn e Zn, são conhecidos por sua atuação eficiente no combate de patogêneses tanto nas plantas como nos seres humanos. O Zn atua na proteção da membrana contra danos oxidativos, proporcionando o funcionamento normal das células mediadoras da imunidade inespecífica, e o Mn desempenha importante papel na ativação de enzimas responsáveis por biossínteses e metabolismos diversos. ${ }^{4-6}$

A elaboração de tabelas de composição alimentar nacionais ou até mesmo regionais é indispensável no fornecimento de informações para uma dieta balanceada, visto que existem diferenças nos teores dos nutrientes devido a fatores inerentes, ambientais e de processamento. ${ }^{2,3}$

As técnicas analíticas mais utilizadas para quantificação de nutrientes inorgânicos em alimentos são as que envolvem espectrometria atômica. Geralmente, essas técnicas exigem prévia destruição total ou parcial da matéria orgânica presente na amostra. O procedimento oficial de destruição da matéria orgânica, calcinação, requer um tempo relativamente longo para sua execução, além de propiciar perdas dos elementos voláteis, o que tem levado à busca de alternativas mais rápidas. ${ }^{2,3,7}$

O uso da radiação micro-ondas apresenta vantagens relacionadas principalmente à melhor eficiência de decomposição e menores brancos analíticos. Métodos convencionais utilizando o aquecimento de amostras em chapas ou em blocos digestores na presença de ácidos e oxidantes continuam sendo investigados, pois apresentam vantagem de um baixo custo. ${ }^{3}$

Diversos pesquisadores têm desenvolvido e otimizado métodos

*e-mail: korn@ufba.br para quantificação de elementos essenciais e tóxicos em arroz por técnicas espectrométricas, que vão dos mais sofisticados que usam radiação de micro-ondas ${ }^{1,8-10}$ aos métodos convencionais utilizando digestão por via úmida, tais como chapas, blocos digestores e bombas de digestão. . $^{311-17}$

Jayasekera e Freitas ${ }^{18}$ utilizaram a técnica de análise por ativação neutrônica instrumental (INAA) na determinação de Al, As, Br, Cd, $\mathrm{Cl}, \mathrm{Co}, \mathrm{Cr}, \mathrm{Cs}, \mathrm{Cu}, \mathrm{Fe}, \mathrm{Hg}, \mathrm{K}, \mathrm{La}, \mathrm{Mg}, \mathrm{Mn}, \mathrm{Mo}, \mathrm{Na}, \mathrm{Rb}, \mathrm{Sc}, \mathrm{Se}$ e Zn em grãos de arroz e a técnica de fluorescência de raios $\mathrm{X}$ de energia dispersiva (EDXRF) para quantificar $\mathrm{Ca}, \mathrm{P}$ e $\mathrm{S}$ em arroz pulverizado. Ambas dispensam a etapa de decomposição da amostra. Ruiz et al. ${ }^{19}$ propuseram um método empregando XRF por comprimento de onda dispersivo para determinação de cloreto, fósforo e enxofre em amostras de alimentos, entre elas arroz. O tempo total de análise para a determinação dos analitos foi menos que 20 min por amostra e a calibração foi feita usando a técnica de adição de analitos. Recentemente, a combinação da espectrometria de massas com plasma indutivamente acoplado (ICP-MS) com a $\mu$ SR-XRF (Micro Synchrotron Radiation - X-Ray Fluorescence) foi empregada para a quantificação e aquisição de microimagens de elementos traço em amostras de arroz..$^{20}$

A espectrometria de fluorescência de raios X de energia dispersiva é uma técnica não destrutiva que pode ser aplicada diretamente a amostras sólidas, sem exigir preparações complexas. ${ }^{21}$ Embora apresente a desvantagem de não atingir limites de detecção comparáveis aos das técnicas de espectrometria atômica, possui grandes vantagens como simplicidade, segurança, baixo custo, uso mínimo de reagentes e vidrarias, gera pouco ou nenhum resíduo e dispensa a etapa de decomposição da amostra. ${ }^{22,23}$

Outro diferencial da EDXRF em relação a outras técnicas espectroscópicas é a ausência de interferências químicas, visto que envolve a participação de elétrons das camadas mais internas sendo, portanto, insensíveis à forma química em que o elemento se encontra. Entretanto, esta técnica é susceptível à interferência física de matriz que é resultante de amostras pouco homogêneas, a interferência espectral, que pode ser contornada pela escolha de linhas espectrais alternativas e a efeitos de matriz que podem ser 
Tabela 1. Programa de aquecimento para o procedimento de digestão assistida por micro-ondas

\begin{tabular}{|c|c|c|c|c|c|}
\hline Etapa & $\mathrm{Tempo}_{\text {(rampa) }} / \mathrm{min}$ & $\mathrm{Tempo}_{\text {(permanência) }} / \mathrm{min}$ & Potência $_{\text {máx }}$ / W & Temperatura $/{ }^{\circ} \mathrm{C}$ & Pressão / bar \\
\hline 1 & 4 & 2 & 500 & 90 & 35 \\
\hline 2 & 8 & 15 & 750 & 180 & 35 \\
\hline Ventilação & - & 10 & - & - & - \\
\hline
\end{tabular}

corrigidos pelo uso de padrões certificados de composição similar às amostras. ${ }^{24}$

Neste trabalho foi investigado e proposto um método alternativo, que dispensa a etapa de decomposição da amostra, para determinação de manganês e zinco em amostras de arroz empregando a espectrometria de fluorescência de raios $\mathrm{X}$ de energia dispersiva.

\section{PARTE EXPERIMENTAL}

\section{Reagentes e soluções}

Todas as soluções foram preparadas empregando reagentes de grau analítico e água ultrapura, com resistividade específica de 18,2 $\mathrm{M} \Omega \mathrm{cm}^{-1}$, de um sistema de purificação Milli-Q ${ }^{\circledR}$ (Millipore, Bedford, MA, USA). Ácido nítrico concentrado (Merck, Alemanha) e peróxido de hidrogênio 30\% v/v (Merck, Alemanha) foram utilizados. As soluções analíticas de referência foram preparadas a partir da diluição adequada das soluções estoque contendo $1000 \mathrm{mg} \mathrm{L}^{-1}$ de $\mathrm{Mn}$ e Zn (Merck, Alemanha).

\section{Amostras e material de referência certificado}

Foram adquiridas amostras de arroz polido, parboilizado polido, parboilizado integral e integral comercializadas na cidade de Salvador, BA. Foi utilizado o material de referência certificado de farinha de arroz 1568a adquirido do NIST (National Institute of Standards and Technology).

\section{Procedimentos e instrumentação}

As amostras foram quarteadas e uma subamostra pré-tratada. O pré-tratamento constou de seleção, moagem, homogeneização, compactação e acondicionamento.

\section{Moagem}

Uma massa de $15 \mathrm{~g}$ de cada amostra foi moída durante 2 min em um moinho TI-100 C.M.T. Company LTD e acondicionada em frascos plásticos.

\section{Preparo das pastilhas}

Aproximadamente $2 \mathrm{~g}$ de amostra pulverizada foram pesadas em uma balança semianalítica Shimadzu BL $3200 \mathrm{H}$ e compactadas sob pressão de $150 \mathrm{kN}$ durante $30 \mathrm{~s}$ em uma prensa Shimadzu MP-35.

As pastilhas foram acondicionadas em sacos plásticos e armazenadas em dessecador. Todas apresentaram aparência firme e compacta, de forma a poderem ser reutilizadas posteriormente. As pastilhas preparadas com a amostra certificada foram manipuladas de forma cuidadosa para evitar que se partissem, pois se trata de um material com baixo teor de umidade.

\section{Quantificação dos analitos}

A determinação dos analitos foi feita empregando espectrômetro de fluorescência de raios X de energia dispersiva Shimadzu EDX900 equipado com tubo de raios X de Rh. As condições operacionais selecionadas foram colimador - $10 \mathrm{~mm}$, atmosfera - ar, tensão do tubo - $50 \mathrm{kV}$, corrente no tubo - $1000 \mu \mathrm{A}$, tempo de irradiação - 100 s, modo de aquisição - Quantitative/FP, linha analítica - K $\alpha$.

As curvas de calibração foram construídas com seis pastilhas escolhidas dentre as amostras. A padronização dessas amostras foi feita mediante digestão em forno de micro-ondas com cavidade em um equipamento modelo Ethos EZ (Milestone, Sorisole, Itália) e quantificação dos analitos em um espectrômetro de massas com plasma indutivamente acoplado ICP-MS Xseries II (Thermo, Germany).

\section{Caracterização dos padrões de calibração}

\section{Procedimento de digestão assistida por micro-ondas}

Pesaram-se, diretamente nos frascos reacionais, massas aproximadas de $250 \mathrm{mg}$ de amostra pulverizada em uma balança analítica BL 210S Sartorius (Alemanha). Adicionaram-se 7,0 mL de $\mathrm{HNO}_{3}$ concentrado e $1,0 \mathrm{~mL}$ de $\mathrm{H}_{2} \mathrm{O}_{2}$ concentrado. Fecharam-se os frascos e executou-se o programa de aquecimento apresentado na Tabela 1. Após resfriamento os frascos reacionais foram abertos na capela, os digeridos e brancos analíticos foram transferidos para frascos volumétricos e os volumes ajustados a 20,0 mL com água ultrapura.

\section{Atribuição dos teores de Mn e Zn}

Um espectrômetro de massas com plasma indutivamente acoplado ICP-MS Xseries II (Thermo, Germany), cuja configuração empregou um nebulizador concêntrico padrão acoplado a uma câmara de nebulização Peltier com pérola de impacto e tocha de quartzo de parte única com injetor de $1,5 \mathrm{~mm}$ e interface de cones Xs, foi utilizado para determinação de Mn e Zn. As condições de operação escolhidas foram potência incidente - $1300 \mathrm{~W}$, vazão do gás do plasma - 13 $\mathrm{L} \min ^{-1}$, vazão do gás do nebulizador - $0,89 \mathrm{~L} \min ^{-1}$, vazão do gás auxiliar - $0,80 \mathrm{~L} \mathrm{~min}^{-1}$, tempo por isótopo - $20 \mathrm{~ms}$, tempo de leitura e da lavagem - $40 \mathrm{~s}$, isótopos $-{ }^{55} \mathrm{Mn}$ e ${ }^{66} \mathrm{Zn}$.

\section{RESULTADOS E DISCUSSÃO}

\section{Caracterização dos padrões}

Os dados obtidos para o material de referência de farinha de arroz 1568a (NIST) na etapa de caracterização dos padrões são apresentados na Tabela 2. Os valores médios e a estimativa do desvio padrão obtidos das triplicatas da amostra certificada foram concordantes com os valores certificados. O t-teste pareado revelou que não há diferença significativa entre os valores certificados e obtidos ao nível de confiança de $95 \%$.

Tabela 2. Comparação entre valores certificados e obtidos $(n=3)$ para caracterização dos padrões de calibração

\begin{tabular}{|c|c|c|}
\hline Elemento/(mg kg $\left.{ }^{-1}\right)$ & Valor certificado & Valor encontrado \\
\hline $\mathrm{Mn}$ & $20,0 \pm 1,6$ & $18,4 \pm 0,3$ \\
\hline $\mathrm{Zn}$ & $19,4 \pm 0,5$ & $20,5 \pm 3,6$ \\
\hline
\end{tabular}

\section{Validação do método proposto}

Os parâmetros de desempenho avaliados para o método proposto 
Tabela 3. Parâmetros de desempenhos do método proposto

\begin{tabular}{|c|c|c|c|c|}
\hline Elementos $/\left(\mathrm{mg} \mathrm{kg}^{-1}\right)$ & Faixa Linear & Equação da curva analítica & LOD & LOQ \\
\hline $\mathrm{Mn}$ & $4,2-33,1$ & $\mathrm{I}=0,0053+8,1938 \times 10^{-4}[\mathrm{Mn}]$ & 5,1 & 17,1 \\
\hline $\mathrm{Zn}$ & $9,9-104,2$ & $\mathrm{I}=-0,0226+0,0029[\mathrm{Zn}]$ & 2,2 & 7,4 \\
\hline
\end{tabular}

foram limite de detecção (LOD), limite de quantificação (LOQ), linearidade, precisão e exatidão.

\section{Limites de detecção e quantificação}

Os limites de detecção e de quantificação foram obtidos a partir de 11 análises dos padrões das curvas de calibração com menor concentração de cada elemento. ${ }^{2}$ Os valores calculados pelo método baseado em parâmetros da curva analítica estão apresentados na Tabela 3.

\section{Linearidade}

Os resultados das curvas analíticas estão apresentados resumidamente na Tabela 3. Os coeficientes de correlação obtidos foram superiores a 0,99 , o que implica na linearidade das curvas, conforme condições recomendadas pela ANVISA e INMETRO..$^{25}$

\section{Exatidão}

Para avaliar a exatidão do procedimento proposto foram analisadas cinco pastilhas preparadas com a amostra certificada de farinha de arroz (1568a NIST). Os resultados mostrados na Tabela 4 são concordantes entre si. O t-teste pareado revelou que não há diferença significativa entre os valores certificados e obtidos ao nível de confiança de $95 \%$.

Tabela 4. Resultados obtidos para Mn e Zn no material de referência NIST $1568 \mathrm{a}(\mathrm{n}=5)$

\begin{tabular}{ccc}
\hline Elemento/( $\left.\mathrm{mg} \mathrm{kg}^{-1}\right)$ & Valor certificado & Valor encontrado \\
\hline $\mathrm{Mn}$ & $20,0 \pm 1,6$ & $22,1 \pm 1,6$ \\
$\mathrm{Zn}$ & $19,4 \pm 0,5$ & $21,5 \pm 1,6$ \\
\hline
\end{tabular}

\section{Precisão}

Para determinação da precisão foram preparadas 11 pastilhas da amostra certificada e efetuadas 3 medições em cada pastilha. Conforme mostrado na Tabela 5, uma boa precisão foi alcançada, com desvio padrão relativo de, respectivamente, 3,5 e 5,0\% para Zn e Mn.

Tabela 5. Teste de repetibilidade $(n=11)$

\begin{tabular}{ccccc}
\hline $\begin{array}{c}\text { Elemento/ } \\
\left(\mathrm{mg} \mathrm{kg}^{-1}\right)\end{array}$ & Média & $\begin{array}{c}\text { Valor } \\
\text { mínimo }\end{array}$ & $\begin{array}{c}\text { Valor } \\
\text { máximo }\end{array}$ & $\begin{array}{c}\text { RSD } \\
(\%)\end{array}$ \\
\hline $\mathrm{Mn}$ & $21,3 \pm 1,1$ & 19,0 & 22,4 & 5,0 \\
$\mathrm{Zn}$ & $21,7 \pm 0,8$ & 20,5 & 22,6 & 3,5 \\
\hline
\end{tabular}

\section{Aplicação do método}

O método proposto foi aplicado para determinação de Mn e Zn em amostras de arroz adquiridas em supermercados da cidade de Salvador - BA. Foram analisadas 18 amostras. As concentrações médias obtidas são mostradas na Tabela 6 .

Na Figura 1 são mostrados os espectros de fluorescência de raios $\mathrm{X}$ de amostras de arroz polido, parboilizado polido, parboilizado integral e integral, com as linhas e/ou bandas de emissão característica e as linhas e/ou bandas de espalhamento da fonte.

Os resultados obtidos são concordantes com as faixas de concentração descritas na literatura. ${ }^{26}$
Tabela 6. Composição mineral, em mg kg-1 , de amostras de arroz submetidas a diferentes processos de beneficiamento

\begin{tabular}{lcc}
\hline Subgrupo & $\mathrm{Mn}$ & $\mathrm{Zn}$ \\
\hline Polido & $9,4 \pm 0,3$ & $19,6 \pm 0,5$ \\
$6,7 \pm 0,4$ & $19,9 \pm 1,1$ \\
$14,1 \pm 1,1$ & $21,5 \pm 0,5$ \\
$14,1 \pm 1,1$ & $19,4 \pm 0,7$ \\
$13,4 \pm 0,8$ & $21,1 \pm 1,0$ \\
Parboilizado polido & $6,8 \pm 1,9$ & $12,7 \pm 1,1$ \\
& $6,0 \pm 0,5$ & $13,3 \pm 0,7$ \\
& $7,3 \pm 0,6$ & $14,2 \pm 1,0$ \\
& $6,3 \pm 0,7$ & $14,7 \pm 0,7$ \\
& $7,4 \pm 0,5$ & $14,7 \pm 0,6$ \\
& $8,0 \pm 0,7$ & $14,0 \pm 0,4$ \\
Integral & $6,1 \pm 0,3$ & $14,3 \pm 0,4$ \\
& $7,2 \pm 0,6$ & $14,7 \pm 1,3$ \\
& $6,1 \pm 0,4$ & $15,2 \pm 0,8$ \\
& $7,4 \pm 0,6$ & $13,0 \pm 0,8$ \\
& $31,2 \pm 2,4$ & $20,3 \pm 0,8$ \\
& $26,8 \pm 1,7$ & $18,9 \pm 1,1$ \\
& $44,5 \pm 1,8$ & $24,7 \pm 0,7$ \\
\hline & & \\
& &
\end{tabular}

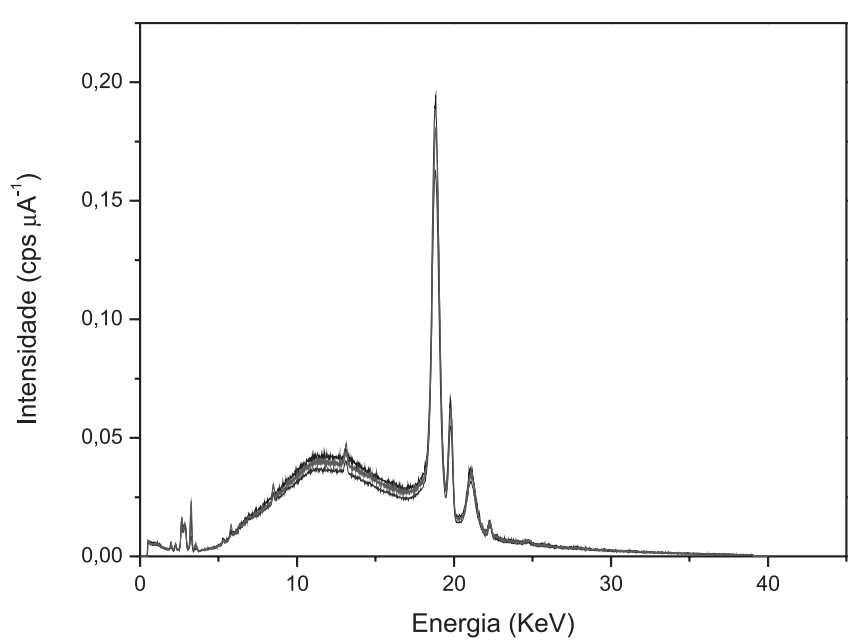

Figura 1. Espectros superpostos de amostras de arroz polido, parboilizado polido, parboilizado integral e integral

\section{CONCLUSÕES}

Neste trabalho foi proposto um método alternativo para determinação de $\mathrm{Mn}$ e $\mathrm{Zn}$ em amostras de arroz na forma de pastilhas, empregando a espectrometria de fluorescência de raios X de energia dispersiva (EDXRF). Avaliou-se a calibração com pastilhas de arroz tornadas padrões mediante caracterização por digestão em forno de micro-ondas e quantificação dos analitos por ICP-MS. As curvas 
analíticas obtidas foram lineares com coeficientes de correlação superiores a 0,99 .

O método proposto apresenta vantagens, tais como simplicidade, segurança, baixo custo, redução do tempo de análise, eliminação do uso de reagentes e geração de resíduos e eliminação da etapa de decomposição da amostra, além de apresentar parâmetros de desempenho satisfatórios.

\section{AGRADECIMENTOS}

Ao Conselho Nacional de Desenvolvimento Científico e Tecnológico $(\mathrm{CNPq})$ pelas bolsas concedidas e também à Fundação de Amparo à Pesquisa do Estado da Bahia (FAPESB) e Petróleo Brasileiro S. A. (PETROBRÁS) pelos financiamentos obtidos.

\section{REFERÊNCIAS}

1. Heinemann, R. J. B.; Fagundes, P. L.; Pinto, E. A.; Penteado, M. V. C.; Lanfer-Marquez, U. M.; J. Food Comp. Anal. 2005, 18, 287.

2. AOAC; Official Methods of Analysis, $17^{\text {th }}$ ed., Association of Official Analytical Chemist: Washington D.C., 2000.

3. Okada, I. A.; Duran, M. C.; Buzzo, M. L.; Dovidauskas, S.; Sakuma, A. M.; Zenebon, O.; Ciênc. Tecnol. Aliment. 2007, 27, 492.

4. Dordas, C.; Agron. Sustain. Dev. 2008, 28, 33.

5. Shankar, A. H.; Prasad, A. S.; Am. J. Clin. Nutr. 1998, 68, 447S.

6. Mohammed, N. K.; Spyrou, N. M.; J. Radioanal. Nucl. Chem. 2009, 281, 79 .

7. Santos, W. P. C.; Gramacho, D. R.; Teixeira, A. P.; Costa, A. C. S.; Korn, M. G. A.; J. Braz. Chem. Soc. 2008, 19, 1.

8. Jorhem, L.; Åstrand, C.; Sundström, B.; Baxter, M.; Stokes, P.; Lewis, J.; Peterson Grawé, K.; Food Addit. Contam. 2008, 25, 284.

9. Saygi, K. O.; Melek, E.; Tuzen, M.; Soylak, M.; Talanta 2007, 71, 1375.
10. Nardi, E. P.; Evangelista, F. S.; Tormen, L.; Saint Pierre, T. D.; Curtius, A. J.; Souza, S. S. De; Barbosa Jr, F.; Food Chem. 2009, 112, 727.

11. Schoof, R. A.; Yost, L. J.; Eickhoff, J.; Crecelius, E. A.; Cragin, D. W.; Meacher, D. M.; Menzel, D. B.; Food Chem. Toxicol. 1999, 37, 839.

12. Schuhmacher, M.; Domingo, J. L.; Llobet, J. M.; Corbella, J.; Bull. Environ. Contam. Toxicol. 1994, 53, 54.

13. Mateos, C. J.; Aguilar, M. V.; Martìnez-Para, M. C.; Eur. Food Res. Technol. 2005, 220, 42

14. Zhang, Z. W.; Moon, C. S.; Watanabe, T.; Shimbo, S.; Ikeda, M.; Biol. Trace Elem. Res. 1997, 57, 39.

15. Hu, Q.H.; Chen, L.C.; Xu, J.; Zhang, Y. L.; Pan, G. X.; J. Sci. Food Agric. 2002, 82, 869 .

16. Chamon, A. S.; Gerzabek, M. H.; Mondol, M. N.; Ullah, S. M.; Rahman, M.; Blum, W. E. H.; Commun. Soil Sci. Plant Anal. 2005, 36, 889.

17. Demirbas, A.; Food Chem. 2005, 90, 773.

18. Jayasekera, R.; Freitas, M. C.; Biol. Trace Elem. Res. 2005, 103, 83.

19. Ruiz, T. P.; Cordoba, M. H.; Gonzalez, R. O.; J. Assoc. Offic. Anal. Chem. 1991, 74, 625.

20. Mihucz, V. G.; Silversmit, G.; Szalóki, I.; Samber, B.; Schoonjans, T.; Tatár, E.; Vincze, L.; Virág, I.; Yao, J.; Záray, G.; Food Chem. 2010, 121, 290.

21. Schimidt, F.; Bueno, M. I. M. S.; Poppi, R. J.; Quim. Nova 2002, 25, 949.

22. Pataca, L. C. M.; Bortoleto, G. G.; Bueno, M. I. M. S.; Quim. Nova 2005, 28, 579.

23. Bueno, M. I. M. S.; Castro, M. T. P. O.; Souza, A. M.; Oliveira, E. B. S.; Teixeira, A. P.; Chemom. Intell. Lab. Syst. 2005, 78, 96.

24. Nagata, N.; Bueno, M. I. M. S.; Peralta-Zamora, P. G.; Quim. Nova 2001, 24, 531.

25. Ribani, M.; Bottoli, C. B. G.; Collins, C. H.; Jardim, I. C. S. F.; Melo, L. F. C.; Quim. Nova 2004, 27, 771.

26. Walter, M.; Marchezan, E.; Avila, L. A.; Ciência Rural 2008, 38, 1184. 